in hospital and profound long term health consequences. It charts a journey to a more effective model of treatment and self-management. This now sees her largely symptom free, on minimal lupus medication (blood pressure tablets) and leading an active personal and professional lifestyle.

Mary draws from her four decade experience to suggest a model of doctor - patient communication, collaboration and partnership that has implications for improving outcomes and quality of life for all lupus patients.

Methods

- Giving the patient a voice. Understanding the powerful psychological benefits with consequential direct and indirect physical benefits for the patient when they are regarded as an "equal partner", an active as opposed to a passive participant in the treatment journey.

- Importance of information sharing with the patient, the treating immunologist and the general practitioner.

- Understanding that the fluctuating and multi-organ nature of lupus symptoms means that treating obvious presenting symptoms without understanding and treating the underlying auto-immune causes can and does lead to treatment errors and adverse patient outcomes.

- Addressing the whole patient, their physical, mental and emotional wellbeing, and the general hormonal system can greatly reduce ongoing symptoms and acute flares.

Results Improved patient outcomes.

Conclusions A more effective model of care.

\section{THE ITALIAN SLE SURVEY BY WEB: INVESTIGATING PATIENTS' UNMET NEEDS WITH ONLINE SURVEY TOOLS}

M falanga*. GRUPPO italiano LES - onlus, research, rome, Italy

10.1136/lupus-2017-000215.146

Background and aims Chronical illness perception and difficulties in everyday life due to disease and medications were explored by the first italian ONLINE SLE SURVEY, designed to assess chronic pain impact and health-care provision quality in Italy.

Methods Online questionnaire was created through Qualtrics, setting geographic data, disease duration, age at diagnosis, comorbidity, disability degree, care practices, treatments, subjective incidence and characteristics of pain. SLE patients were advertised by social media. Participation was voluntary and anonymous.

Results 550 SLE patients provided complete data; F 94.7\%, M 5.3\%; mean age 33 y. (14-82 y.); first SLE diagnosis at mean age 29 y.: 84\% received SLE diagnosis between 18-42 y.; 36\% comorbidity with other (1-6) autoimmune conditions. SLE impact on life is relevant, with specific problems and needs at different disease stage. Stress of life: relevant and worsening illness conditions; frequent relational problems. Need for psychological support: 54\%. Osteoarticular pain is main symptom condition (83\%), but only 54\% use drugs for pain control. Physicians seem not responding to patients" request to take into account impact of pain. Women workers face many difficulties due to many combined factors which severely reduce access to proper care

Conclusions Health-care current model doesn't allow respect of SLE patients complex needs: most remain dissatisfied, affecting quality of life and doctor-patient concordance. SLE SURVEY highlights importance of competent clinical listening by physicians and capacity to hold patient's crisis. GRUPPO ITALIANO LES - a volunteer patients' organisation - established ONLINE SLE SURVEY practice to explore current facets, development of patients' needs, and set social-health policies

\section{A CASE OF DERMATOMYOSITIS WITH ELEVATED SERUM KL-6 LEVEL ASSOCIATED WITH OVARIAN CANCER}

${ }^{1} \mathrm{~T}$ Katagiri* ${ }^{*}{ }^{1} \mathrm{H}$ Ito, ${ }^{1} \mathrm{Y}$ Fujisawa, ${ }^{1} \mathrm{~K}$ Mizushina, ${ }^{1} \mathrm{~S}$ Takenaka, ${ }^{1} \mathrm{H}$ Ayako, ${ }^{1} \mathrm{~T}$ Ogura, ${ }^{2} \mathrm{~S}$ Nagasaki, ${ }^{2} \mathrm{~S}$ Komiyama, ${ }^{3} \mathrm{~T}$ Oharaseki, ${ }^{1} \mathrm{H}$ Kameda. ${ }^{1}$ Division of RheumatologyDepartment of Internal Medicine, Toho University Ohashi Medical Cener, Meguro-ku, Japan; ${ }^{2}$ Department of Gynaecology, Toho University Ohashi Medical Cener, Meguro-ku, Japan; ${ }^{3}$ Department of Pathology, Toho University Ohashi Medical Cener, Meguro-ku, Japan

\subsection{6/lupus-2017-000215.147}

Background and aims Elevated serum Krebs von den Lungen-6 (KL-6) is a biomarker of interstitial pneumonia and some types of cancers. Because patients with dermatomyositis (DM) may accompany interstitial pneumonia and malignancies, serum KL-6 level has been commonly examined for patients with DM in Japan.

Methods A 62-year-old woman developed myalgia in bilateral thighs in November 2014. She was admitted to our hospital in the following month because of progressive bilateral proximal muscle weakness and myalgia, elevated serum creatine kinase (CK: $5569 \mathrm{U} / \mathrm{L})$, and Gottron's papule. Although muscle biopsy had not been done, the diagnosis as DM was further supported by muscle MRI, electromyogram and skin biopsy. The body CT revealed pelvic mass with ascites, but not interstitial pneumonia.

Results Although prednisolone $50 \mathrm{mg} /$ day and intravenous immunoglobulin therapy against progressive muscle weakness including severe dysphagia only showed a partial response, the surgical resection of ovarian mass, identified as ovarian serous adenocarcinoma stage II c, followed by chemotherapy resulted in clinical remission of DM. Serum KL-6 level, as well as CA125 decreased below the upper normal limit.

Conclusions The present case suggests that the measurement of serum KL-6 may be useful in the evaluation of patients with DM.

\section{THE ENGAGEMENT OF SLE PATIENTS IN THEIR HEALTH CARE}

${ }^{1} \mathrm{D}$ Mazzoni, ${ }^{2} \mathrm{~A}$ Cornet ${ }^{*},{ }^{2} \mathrm{~K}$ Myllys, ${ }^{2} \mathrm{~B}$ van Leeuw, ${ }^{1} \mathrm{E}$ Cicognani. ${ }^{1}$ University of Bologna, Department of Psychology, Bologna, Italy; ${ }^{2}$ Lupus Europe, Lupus Europe, Romford, UK

\subsection{6/lupus-2017-000215.148}

Background and aims Patient engagement is recognised as a crucial component of high-quality healthcare services. Among rheumatic diseases, Systemic Lupus Erythematosus (SLE) appears particularly challenging for the engagement of patients in their own care. According to the Patient Health Engagement (PHE) model, patient engagement is a dynamic phenomenon that proceeds through four experiential positions (blackout, arousal, adhesion, and eudaimonic project). The aim of the present study was to describe the engagement process through the experiences of SLE patients.

Methods Ten in-depth interviews and four fous group were conducted with an international sample of SLE patients from different European countries. Interviews focused on several 